\title{
Investigation of the molecular mechanisms underlying postoperative recurrence in prostate cancer by gene expression profiling
}

\author{
CHENG YAJUN $^{1 *}$, TANG YUAN ${ }^{2 *}$, WANG ZHONG ${ }^{1}$ and XU BIN ${ }^{1}$ \\ ${ }^{1}$ Department of Urology, Shanghai Ninth People's Hospital, Shanghai Jiaotong University School of Medicine, \\ Shanghai 200011; ²Department of Gastrointestinal Surgery, Changzheng Hospital, \\ Second Military Medical University, Shanghai 200003, P.R. China
}

Received March 16, 2017; Accepted October 20, 2017

DOI: $10.3892 /$ etm.2017.5510

\begin{abstract}
The present study aimed to identify potential genes associated with prostate cancer $(\mathrm{PCa})$ recurrence following radical prostatectomy $(\mathrm{RP})$ in order to improve the prediction of the prognosis of patients with PCa. The GSE25136 microarray dataset, including 39 recurrent and 40 non-recurrent $\mathrm{PCa}$ samples, was downloaded from the Gene Expression Omnibus database. Differentially-expressed genes (DEGs) were identified using limma packages, and the pheatmap package was used to present the DEGs screened using a hierarchical cluster analysis. Furthermore, gene ontology functional enrichment analysis was used to predict the potential functions of the DEGs. Subsequently, Kyoto Encyclopedia of Genes and Genomes pathway enrichment analyses were performed to analyze pathway enrichment of DEGs in the regulatory network. Lastly, a protein-protein interaction (PPI) network of the DEGs was constructed using Cytoscape software to understand the interactions between these DEGs. A total of 708 DEGs were identified in the recurrent and non-recurrent PCa samples. Functional annotation revealed that these DEGs were primarily involved in cell adhesion, negative regulation of growth, and the cyclic adenosine monophosphate and mitogen-activated protein kinase (MAPK) signaling pathways. Furthermore, five key genes, including cluster of differentiation 22, insulin-like growth factor-1, inhibin $\beta$ A subunit, MAPK kinase 5 and receptor tyrosine kinase like orphan receptor 1 , were identified through PPI network analysis.
\end{abstract}

Correspondence to: Professor Xu Bin, Department of Urology, Shanghai Ninth People's Hospital, Shanghai Jiaotong University School of Medicine, 639 Zhizaoju Road, Shanghai 200011, P.R. China E-mail: chxb2004@126.com

*Contributed equally

Key words: prostate cancer, recurrence, differentially-expressed genes, IGF-1
The results of the present study have provided novel ideas for predicting the prognosis of patients with PCa following RP.

\section{Introduction}

Prostate cancer ( $\mathrm{PCa}$ ), as one of the most common men's malignancy in America, is the second leading cause of cancer-related death in men (1). Although more than $80 \%$ of PCa was diagnosed as localized disease and commonly treated by radical prostatectomy (RP), postoperative recurrence occurred in about $15 \%$ of patients within 5 years and up to $40 \%$ within 10 years (2). Recurrence of localized PCa following treatment can lead to lethal metastatic castration-resistant PCa. Various biomarkers have been reported for PCa recurrence surveillance, including preoperative prostate specific antigen (PSA) value, Gleason score, lymph node invasion and others, but not cancer-specific and inaccurate (3). Therefore, more efforts should be devoted for identifying disease specific markers of PCa recurrence that can better directly offer practical aid to drug treatment and lead to improved survival and reductions in morbidity.

Although the mechanism underlying $\mathrm{PCa}$ is not yet completely understood, multiple genes to help predict $\mathrm{PCa}$ risk have been proposed by considerable researches. Brian R. $\mathrm{Hu}$ et al (4) reported that AXIN2 expression could not only predict PCa recurrence, but also promoted tumor growth and metastasis in vivo and vitro. Hao et al (5) found that XPO6 expression was elevated in $\mathrm{PCa}$ and maybe a potential prognostic biomarker for PCa recurrence. Additionally, some other targets from blood and (or) urine have been examined and identified, including KLK2-KLK3 SNP rs2735839, 17p12 SNP rs4054823 and Eotaxin-1 (6,7). However, few of these profiles have been adopted in the clinic after RP to predict recurrence $\mathrm{PCa}$. Therefore, there is still a need for novel tumor biomarkers that can help improve prediction of prostate cancer recurrence upon clinical variables.

To explore more meaningful molecular biomarker for predicting the prostate cancer prognosis, technologies with high-throughput screen was implied to identify the genes. Microarray data GSE 25136 with 39 recurrent and 40 non-recurrent PCa was published and analyzed by Stephenson et al (8) 
via leave-one-out-cross-validation (LOOCV) approach and the results showed that Etoposide-induced 2.4 mRNA (EI24) and mitogen-activated protein kinase kinase kinase kinase 4 (MAP4K4) were the most highly overexpressed genes and erythrocyte membrane protein band 4.9 (EPB49) was the most highly underexpressed gene in recurrent tumors compared with primary PCa and may be the potential biomarker. Subsequently, Sun and Goodison (9) conducted a more advanced computational algorithm to analyze the Microarray data GSE25136 and acquire more accurate biomarkers for predicting the prognosis of PCa. With technological development, bioinformatics has been a mainstream tool to analyze the microarray data. In the present study, microarray data GSE25136 $(8,9)$ was employed to identify differentially expressed genes (DEGs) between $\mathrm{PCa}$ and $\mathrm{PCa}$ recurrence samples with Limma package in $\mathrm{R}$ language. Furthermore, gene ontology (GO) and pathway enrichment analysis was performed to screen the DEGs. Lastly, PPI networks of DEGs was constructed by Cytosacpe mapping software and hub genes was identified by the STRING database. Therefore, it is better for us to further understand the molecular mechanisms of PCa.

\section{Materials and methods}

Microarray data. The gene expression profiles of GSE25136 were downloaded from the GEO database. GSE25136 based on Affymetrix GPL96 platform (Affymetrix Human Genome U133A Array), was submitted by Sun and Goodison (9) and updated on Jul 01, 2016. The GSE25136 dataset contained 79 PCa samples treated by radical prostatectomy (RP) in 1993 and 1999, including 39 recurrent and 40 non-recurrent $\mathrm{PCa}$ samples. When serum level of PSA consecutively increased at least 3 times post operation, the patients were classified as disease recurrence; non-recurrent patients with an undetectable PSA $(<0.05 \mathrm{ng} / \mathrm{ml})$ for at least 5 years after RP were identified. The clinical characteristics of all 79 patients has been completely described by Stephenson et al (8). In briefly, Median PSA level and Prostatectomy Gleason sum of patients with recurrence were higher than those in non-recurrenct $\mathrm{PCa}$ patients, and the number of patients with Extracapsular extension, positive surgical margins, seminal vesicle invasion were greater in recurrent $\mathrm{PCa}$ group compared with non-recurrent PCa group.

Identification of DEGs. The raw data files used for the analysis included cell files (Affymertix platform). The data was preprocessed by $\mathrm{R}$ biocondutor RMA Packages, and DEGs were identified by limma packages in recurrent $\mathrm{PCa}$ compared with non-recurrent PCa samples. DEGs were identified with a change fold and defined a P-value cutoff of $<0.05$ to be statistically significant. Hierarchical clustering analysis was applied to categorize the data into two groups that had similar expression patterns. Heatmap was performed by the pheatmap package analysis with joint between-within distances. Expression values from multiple clones or probe sets mapping to the same Unigene Cluster ID were averaged.

Gene ontology (GO) analysis of DEGs. The Database for Annotation, Visualization and Integrated Discovery (DAVID; https://david.ncifcrf.gov/) provides a comprehensive set of novel and powerful tools for assigning biological meaning to a set of genes (10). The false recovery rate (FDR) $<0.05$ was used as the cut-off criterion for GO functional enrichment analysis by DAVID.

Pathway enrichment analysis of DEGs in the regulatory network. KEGG (http://www.genome.jp/) is acknowledge base for systematic analysis of gene functions, linking genomic information with higher-order functional information (11). $\mathrm{P}<0.05$ was used as the cut-off criterion for the Kyoto Encyclopedia of Genes and Genomes pathway enrichment analysis using DAVID.

Integration of protein-protein interaction (PPI) network and module analysis. The search Tool for the Retrieval of Interacting Genes (STRING) database is an online tool designed to evaluate PPI information. STRING (version 10.0) covers 5,214,234 proteins from 1,133 organisms. To evaluate the interactive relationships between the DEGs, the DEGs were mapped to STRING, and only experimentally validated interactions with a combined score $>0.4$ were selected as significant. Then, the Cytoscape software was used to construct the PPI network. The plug-in Molecular Complex Detection (MCODE) was used to screen the modules of PPI network in Cytoscape with a degree cutoff $=2$, node score cutoff $=0.2$, $\mathrm{k}$-core $=2$, max depth from seed $=100$. The criteria were set as follows: MCODE scores $>4$ and number of nodes $>4$. $\mathrm{P}<0.05$ was considered to have significant differences.

\section{Results}

Identification of DEGs. After data, including 39 recurrent $\mathrm{PCa}$ samples and 40 non-recurrent PCa samples, was downloaded from GEO database and preprocessed, 708 DEGs, including 212 up genes and 496 down genes were identified using limma packages on the basis of the cut-off criteria $(\mathrm{P}<0.05$ and fold control $(\mathrm{FC}) \geq 1.4$ criteria) in recurrent samples compared with non-recurrent samples. Subsequently, DEGs were performed hierarchical clustering analysis and it can accurately classify the prostate samples as recurrent $\mathrm{PCa}$ tissues and non-recurrent $\mathrm{PCa}$ tissues (Fig. 1: left, recurrent $\mathrm{PCa}$; right, non-recurrent $\mathrm{PCa}$ ). Additionally, Top up 50 DEGs and down 50 DEGs expression with most significant was shown in Fig. $1(\mathrm{P}<0.05)$.

GO functional enrichment analysis. In order to gain further insight into the function of the identified DEGs, we uploaded DEGs to the online biological classification software DAVID to identify typical GO categories. Go analysis showed that DEGs were significantly enriched in biological processes (BP), including cell adhesion, negative regulation of growth, extracellular matrix organization, negative regulation of cell migration, apoptotic signaling pathway (Table I). For cell components, DEGs were enriched in focal adhesion, extracellular exosome, cell-cell adherens junction, proteinaceous extracellular matrix (Table I). Finally, Go molecular function analysis showed that DEGs were enriched in protein binding, protein homodimerization activity, cadherin binding involved in cell-cell adhesion, insulin receptor binding (Table I). 
Table I. Gene ontology analysis of differentially expressed genes associated with PCa recurrence.

\begin{tabular}{|c|c|c|c|c|}
\hline Category & Term/gene function & $\begin{array}{l}\text { Gene } \\
\text { count }\end{array}$ & $\%$ & P-value \\
\hline GOTERM_BP_DIRECT & GO:0007155 cell adhesion & 38 & 6.551724138 & $3.82 \mathrm{E}-07$ \\
\hline GOTERM_BP_DIRECT & GO:0045926 negative regulation of growth & 8 & 1.379310345 & $1.33 \mathrm{E}-06$ \\
\hline GOTERM_BP_DIRECT & GO:0030198 extracellular matrix organization & 22 & 3.793103448 & $1.72 \mathrm{E}-06$ \\
\hline GOTERM_BP_DIRECT & GO:0030336 negative regulation of cell migration & 12 & 2.06896552 & $2.57 \mathrm{E}-04$ \\
\hline GOTERM_BP_DIRECT & GO:0097190 apoptotic signaling pathway & 10 & 1.72413793 & 4.71E-04 \\
\hline GOTERM_CC_DIRECT & GO:0005925 focal adhesion & 42 & 7.241379 & 8.83E-12 \\
\hline GOTERM_CC_DIRECT & GO:0070062 extracellular exosome & 149 & 25.68966 & $1.26 \mathrm{E}-11$ \\
\hline GOTERM_CC_DIRECT & GO:0005913 cell-cell adherens junction & 23 & 3.965517241 & 4.87E-04 \\
\hline GOTERM_CC_DIRECT & GO:0005578 proteinaceous extracellular matrix & 23 & 3.965517 & 3.33E-05 \\
\hline GOTERM_MF_DIRECT & GO:0005515 protein binding & 372 & 64.13793103 & $3.67 \mathrm{E}-15$ \\
\hline GOTERM_MF_DIRECT & GO:0042803 protein homodimerization activity & 46 & 7.931034483 & 2.34E-05 \\
\hline GOTERM_MF_DIRECT & GO:0098641 cadherin binding involved in cell-cell adhesion & 20 & 3.448275862 & 0.002756127 \\
\hline GOTERM_MF_DIRECT & GO:0005158 insulin receptor binding & 6 & 1.034482759 & 0.0028733 \\
\hline
\end{tabular}

$\mathrm{BP}$, biological process; $\mathrm{CC}$, cell component; MF, molecular function.

KEGG pathway analysis. We employed KEGG pathway enrichment analysis to identify the most significantly enriched pathways of the DEGs. 10 biological pathways which significant enriched with DEGs including cAMP signaling pathway, MAPK signaling pathway, Adherensjunction, Calcium signaling pathway, Pathways in cancer, Proteoglycans in cancer, Transcriptional misregulation in cancer, Leukocyte transendothelial migration, Focal adhesion and Ras signaling pathway (Table II).

Construction of the PPI network. Cytosacpe mapping software was employed to construct the PPI network of DEGs. A total of 663 nodes and 8,871 edges were analyzed using plug-ins MCODE. The top 5 significant modules with MCODE scores $>4$ and nodes $>4$ in whole network were screened by analysis in the STRING database, and the hub gene in each cluster, also called the seed, was identified by on the basis of the highest modules scoring in the cluster including Insulin-like growth factor-1 (IGF-1) (Fig. 2A), mitogen-activated protein kinase kinase 5 (MAP2K5) (Fig. 2B), Receptor tyrosine kinase like orphan receptor 1 (ROR1) (Fig. 2C), Inhibin beta A (INHBA) (Fig. 2D), and differentiation-22 (CD22) (Fig. 2E). All clusters are named after the hub gene name, and of these clusters, IGF-1 modules showed a highest MCODE scores in whole network, with 20.606. Additionally, IGF-1 modules consisted of 34 nodes and 340 edges; MAP2K5 modules consisted of 43 nodes and 306 edges; ROR1 modules consisted of 71 nodes and 464 edges; INHBA modules consisted of 51 nodes and 119 edges; CD22 modules consisted of 38 nodes and 74 edges.

\section{Discussion}

PCa is the fourth leading global cause of human malignancies worldwide, and is a product of mutation in genomics including cumulative genetic, epigenetic, somatic, and endocrine aberrations (12). Of the differential expression of genes caused by various mutations, some specific genes are positively or negatively associated with therapy resistance and poor outcomes in PCa. The wide application of microarray and high through put sequencing has made it possible to identify the more appropriate genes to predict the prognosis of $\mathrm{PCa}$ after RP from thousands of genes in human genome level (12). In the present study, we extracted the data from GSE25136 and 708 DEGs between recurrent PCa samples and non-recurrent PCa samples using bioinformatics analysis were screened out. Functional annotation showed that these DEGs were mainly involved in cell adhesion, focal adhesion, protein binding, cAMP signaling pathway and MAPK signaling pathway. In addition, to better understand the interaction of these DEGs, a PPI network was constructed and we identified four key genes, including CD22, IGF-1, INHBA, MAP2K5 and ROR1, that can provide new ideas for predict the prognosis in $\mathrm{PCa}$ following RP.

The GO term analysis showed that these DEGs were mainly involved cell adhesion, focal adhesion, and protein binding. In addition, cAMP and MAPK signaling pathway were shown to participate int PCa recurrence by KEGG pathway analysis. Classical signal transduction pathway and cAMP signaling pathway have been extensively studied in the context of carcinogenesis by regulating cellular growth and proliferation. cAMP-dependent protein kinase (PKA), as a critical mediator of cAMP signaling pathway, has been demonstrated that it is overexpressed in PCa and has been examined as a potential biomarker for predicting the outcome of PCa patients (13). Androgens are required for the initiation and the development of PCa via stimulating the AR signaling pathway, and androgen ablation therapies, such as chemical or surgical castration, have become a standard against $\mathrm{PCa}$ (14). There is a highly relevant cross-talk between cAMP and AR signaling pathway in $\mathrm{PCa}$ progression, because not only cAMP and PKA activation may result in the stimulation of AR but androgens can also regulate the activity of PKA (15). In addition, cyclic nucleotide 
Color key

Heatmap
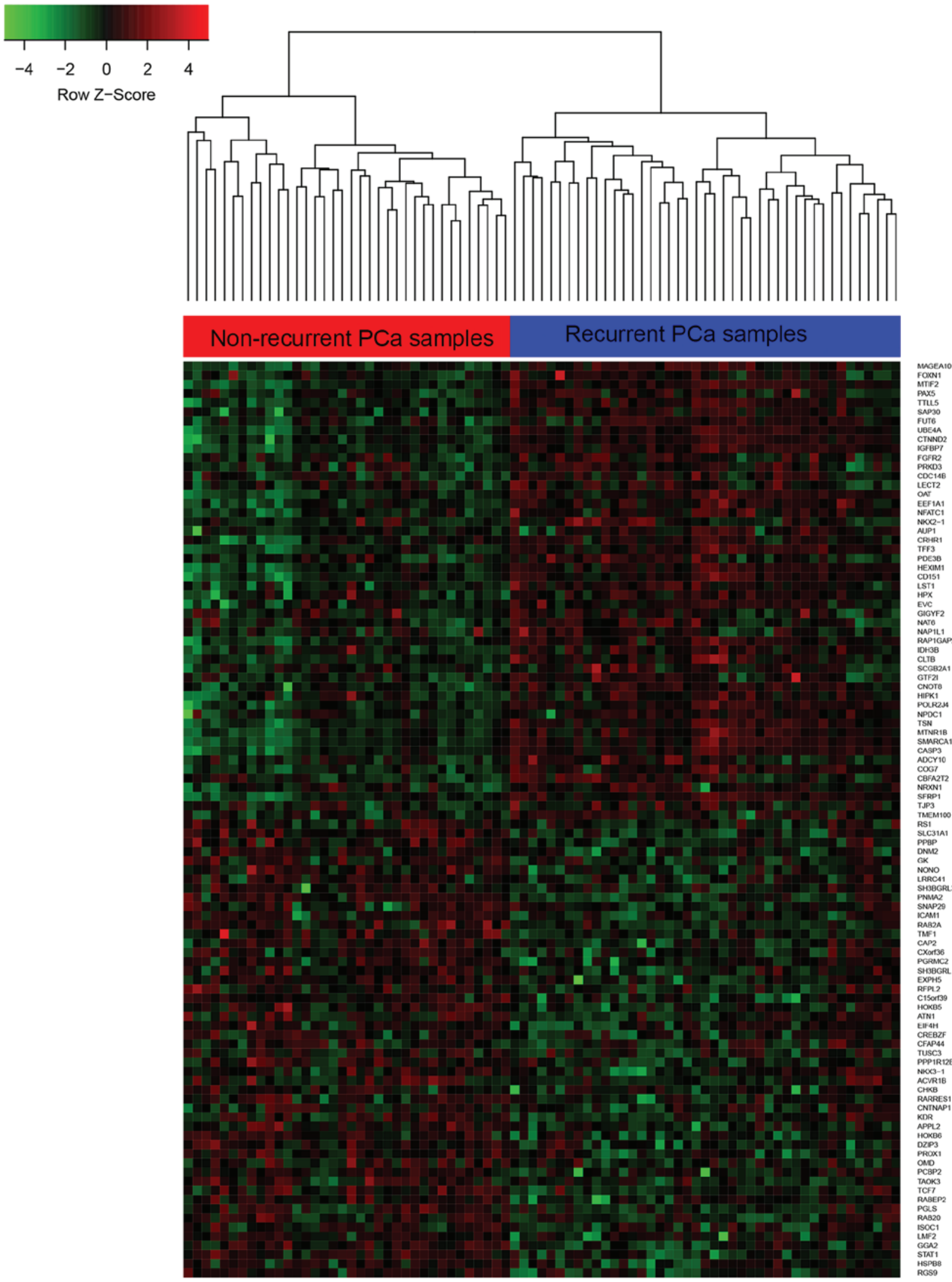

†ำ

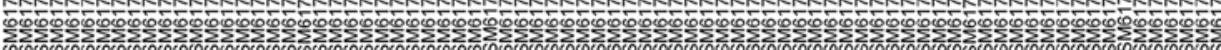

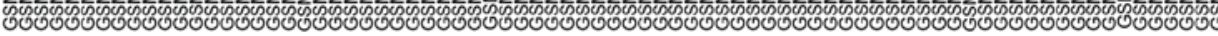

Figure 1. Identification of DEGs between recurrent and non-recurrent prostate cancer tissues by Hierarchical cluster analysis. (Left): Non-recurrent PCa group, (Right): Recurrent PCa group. Each row represents a single gene; each column represents a tissue sample. The gradual color change from green to red represents the changing process from dowregulation to upregulation. 
Table II. KEGG pathway analysis of differentially expressed genes associated with PCa recurrence.

\begin{tabular}{|c|c|c|c|c|c|}
\hline Pathway ID & Name & Gene count & $\%$ & P-value & Genes \\
\hline hsa04024 & cAMP signaling pathway & 23 & 0.021929197 & $5.74 \mathrm{E}-05$ & $\begin{array}{l}\text { PLD1,VAV3,PTGER3,PDE3B, } \\
\text { GRIA3, PDE4D, GABBR2, CNGB1, } \\
\text { BDNF, HTR1A,ATP2B4, NPY, } \\
\text { GRIA2, PDE4A, RAC1, CREB3L2, } \\
\text { RYR2, GNAS, CAMK2B, ADCY10, } \\
\text { FSHB, GLP1R, NFATC1 }\end{array}$ \\
\hline hsa04010 & MAPK signaling pathway & 22 & 0.020975754 & 0.004317491 & $\begin{array}{l}\text { FGFR2, FGF8, FGF7, CACNA1I, } \\
\text { TAOK3, MAPK11, MECOM, } \\
\text { FLNC, FLNB, CDC42, MAP4K4, } \\
\text { CASP3, BDNF, RPS6KA4, PAK2, } \\
\text { SOS1, RAC1, CACNA1G, EGF, } \\
\text { DUSP7, MAP2K5, NFATC1 }\end{array}$ \\
\hline hsa04520 & Adherens junction & 9 & 0.00858099 & 0.012675942 & $\begin{array}{l}\text { ACTB, CDC42, TCF7, CSNK2A1, } \\
\text { BAIAP2, RAC1, SSX2IP, PTPN1, } \\
\text { ACTN3 }\end{array}$ \\
\hline hsa04020 & Calcium signaling pathway & 16 & 0.015255094 & 0.012798992 & $\begin{array}{l}\text { SLC8A2, PTGER3, SPHK2, } \\
\text { SPHK1, CACNA1I, VDAC1, GNAL, } \\
\text { ATP2B4, ATP2A3, RYR3, PDE1A, } \\
\text { CACNA1G, RYR2, GNAS, } \\
\text { CAMK2B, ADRA1D }\end{array}$ \\
\hline hsa05200 & Pathways in cancer & 27 & 0.025742971 & 0.025195199 & $\begin{array}{l}\text { FGFR2, FGF8, TCF7, PTGER3, } \\
\text { CTBP2, FGF7, RXRB, ITGA2, } \\
\text { IGF1, STAT1, MECOM, CDC42, } \\
\text { CASP3, LAMB2, HDAC2, CXCR4, } \\
\text { SOS1, RAC1, MDM2, NKX3-1, } \\
\text { GNAS, GNB3, GNG3, RARB, EGF, } \\
\text { WNT6, APC }\end{array}$ \\
\hline hsa05205 & Proteoglycans in cancer & 16 & 0.015255094 & 0.031484838 & $\begin{array}{l}\text { ACTB, PPP1R12B, ITGA2, IGF1, } \\
\text { MAPK11, FLNC, FLNB, KDR, } \\
\text { CDC42, CASP3, HPSE, SOS1, } \\
\text { RAC1, MDM2, CAMK2B, WNT6 }\end{array}$ \\
\hline hsa05202 & $\begin{array}{l}\text { Transcriptional misregulation } \\
\text { in cancer }\end{array}$ & 14 & 0.013348207 & 0.035045912 & $\begin{array}{l}\text { SUPT3H, FLT1, RXRB, IGF1, } \\
\text { PAX5, GRIA3, GZMB, HDAC2, } \\
\text { REL, LYL1, MDM2, PBX1, } \\
\text { IGFBP3, CDK14 }\end{array}$ \\
\hline hsa04670 & $\begin{array}{l}\text { Leukocyte transendothelial } \\
\text { migration }\end{array}$ & 11 & 0.010487877 & 0.035804774 & $\begin{array}{l}\text { ACTB, CDC42, ICAM1, NOX3, } \\
\text { VAV3, CXCR4, CLDN5, NOX1, } \\
\text { RAC1, MAPK11, ACTN3 }\end{array}$ \\
\hline hsa04510 & Focal adhesion & 16 & 0.015255094 & 0.039374107 & $\begin{array}{l}\text { ACTB, CDC42, FLT1, VAV3, } \\
\text { LAMB2, PAK2, SOS1, PPP1R12B, } \\
\text { RAC1, IGF1, ITGA2, ACTN3, } \\
\text { FLNC, EGF, FLNB, KDR }\end{array}$ \\
\hline hsa04014 & Ras signaling pathway & 17 & 0.016208537 & 0.042199384 & $\begin{array}{l}\text { FGFR2, PLD1, FGF8, FGF7, FLT1, } \\
\text { IGF1, BRAP, KDR, CDC42, PAK2, } \\
\text { REL, SOS1, TEK, RAC1, GNG3, } \\
\text { GNB3, EGF }\end{array}$ \\
\hline
\end{tabular}

$\mathrm{FDR}<0.05$.

phosphodiesterases (PDEs) are involved in the metabolism of cAMP by regulating its degradation (16). PDE4D, as a kind of PDEs, is highly expressed in PCa and has been implicated to promote PCa progression (16). Furthermore, members of the PDE4D subfamily are classified as long, short and super-short.
PDE4D7, as a long isoform member, is downregulated in androgen-independent prostate cancer cells compared with androgen sensitive prostate cancer cells, and inhibited its growth by compartmentalising cAMP (17). R Böttcher et al (18) also showed that it was up-regulated in localized PCa samples 

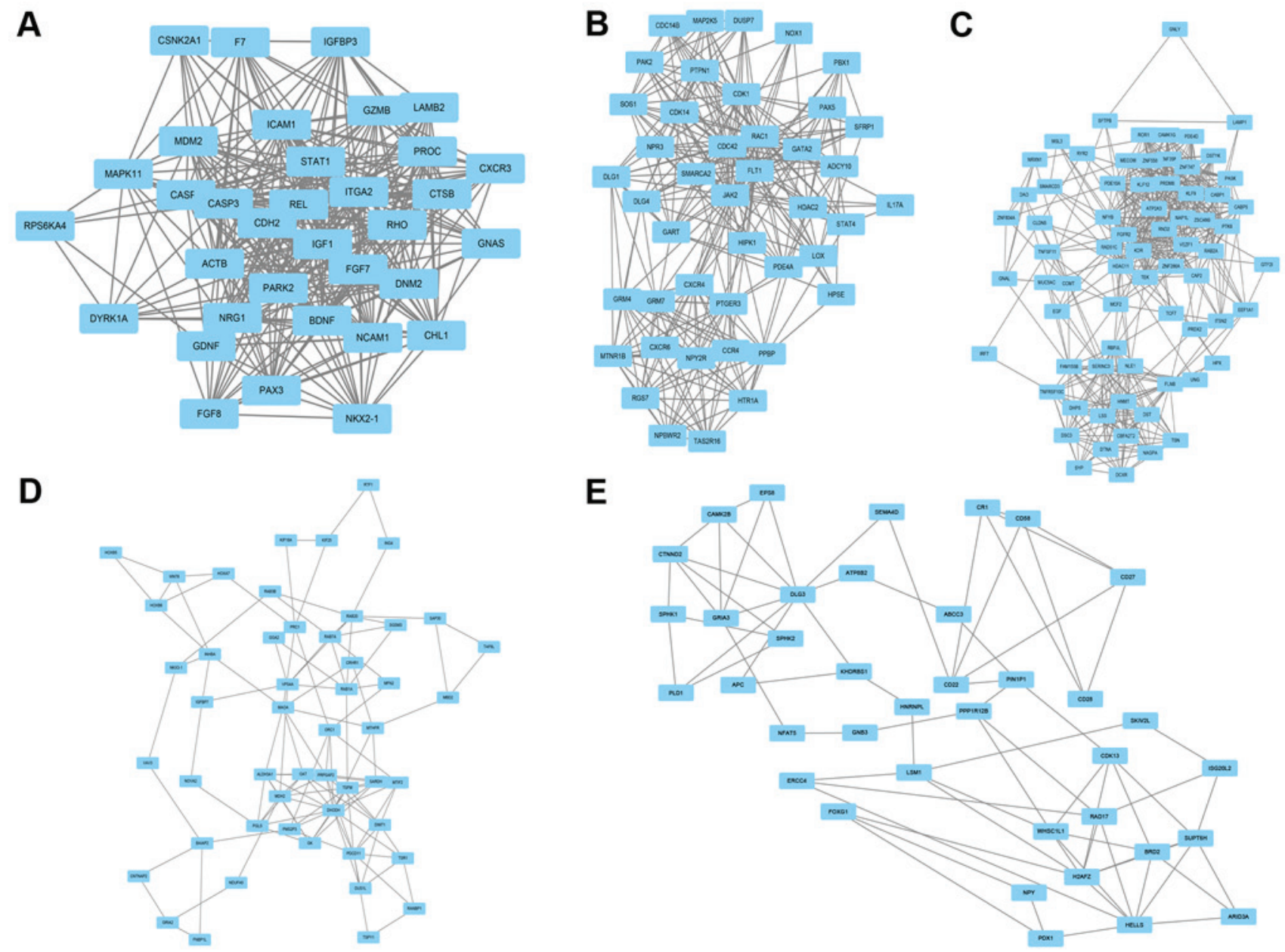

Figure 2. PPI sub-network of hubgenes. (A) IGF-1 modules with 34 nodes and 340 edges; (B) MAP2K5 modules with 43 nodes and 306 edges; (C) ROR1 modules with 71 nodes and 464 edges; (D) INHBA modules with 51 nodes and 119 edges; (E) CD22 modules with 38 nodes and 74 edges in whole network.

compared with the normal adjacent prostate tissues, while its expression diminished with emergence of Castration resistant prostate cancer (CRPC). Other PDE4D isoform composition, such as PDE4D5 and PDE4D9, was also upregulated in PCa and played an import role in PCa progression (19). MAPK signaling pathway also played a vital role in regulating cellular behaviors in response to extracellular stimuli. Dysregulation of p38 MAPK, as a main subgroup of MAPK signaling pathway, are associated with tumor stages and poor survival of $\mathrm{PCa}$ patients (20). The emergence of Castration resistant prostate cancer (CRPC) caused by certain co-activators or through MAPK signaling pathway activities, which lead to the overexpression of anti-apoptotic genes and survival of the cancer cells, thus increasing the PCa related death (21).

Finally, the PPI network with DEGs was constructed and the hub genes exhibiting the highest degree of connectivity were identified, including CD22, IGF-1, INHBA, MAP2K5 and ROR1. IGF-1 was identified as one of the most DEGs in the recurrent PCa samples. IGF-1, also known as somatomedin 1 , is a mitogen that plays a key role in regulating various cell biological behavior, including cell proliferation, differentiation, and apoptosis via endocrine, paracrine and autocrine mechanisms (22). IGF-1 binds to the insulin- like growth factor 1 recep-tor $(\mathrm{IGF}-1 \mathrm{R})$, which is a tyrosine kinase receptor, and initiates a cascade of downstream signal transduction pathways (23). Results published recent studies evaluated the role of IGF-1 in PCa and showed that higher circulating IGF-1 levels were consistently associated with increased risk of $\mathrm{PCa}$ in epidemio-logic studies (24). IGF-1, which is synthesized locally in an autocrine or paracrine manner by PCa cells, may stimulate PCa growth and development (25). Then, IGF has been a pivotal target gene for PCa therapy. Magnolol has been demonstrated that it served as a novel anti-PCa agenet via regulating the expression of IGF-1 in vitro (26). Apigenin effectively suppressed PCa cells growth and metastasis in TRAMP mice by attenuating IGF-I signaling (27). In addition, IGF-1 genotypes and haplotypes were associated with wore survival of PCa patients with bone metastasis (28). Mitogen-activated protein kinase kinase 5 (MAP2K5), also known as MEK5, was overexpressed in $\mathrm{PCa}$, which was associated with tumor metastases and unfavourable survival outcome of $\mathrm{PCa}$ patients (29).

Receptor tyrosine kinase like orphan receptor 1 (ROR1), also known as neurotrophic tyrosine kinase, receptor-related 1 (NTRKR1), is a transmembrane protein belonging to the receptor tyrosine kinase (RTK) family. Down-regulation of ROR1 inhibited human colorectal cancer cell growth and promoted apoptosis (30). ROR1 has been shown to be overexpressed in several solid tumors and its unique expression by malignant cells surface is a target for novel therapeutics, 
especially monoclonal antibodies (mAbs) for the treatment of cancer (31). Inhibin beta A, also known as INHBA, is a subunit of both activin and inhibin, two closely related glycoproteins with opposing biological effects. INHBA is overexpressed in various cancers, including gastric cancer, rothelial carcinoma of the urinary bladder and upper tract and colorectal cancer, demonstrating its association with poor prognosis of patients (32-34). Cluster of differentiation-22 (CD22), as a molecule belonging to the SIGLEC family of lectins, is a transmembrane glycoprotein expressed by mature B cells. Recently, Tuscano and colleagues reported that $\mathrm{CD} 22$, a hallmark marker on B lymphocytes, was expressed on lung cancer cells and might serve as a new target for therapy (35). However, Pop et al (36) reported that the surface of lung cancer cells did not detect CD22 expression, and cannot be killed by anti-CD22 immunotoxins, which have not previously been directly associated with initiation and progression of PCa, according to the present results.

Although data from GSE25136 have been analyzed by previous authors, and some DEGs have been identified, the uniqueness of the present study is that Limma package in $\mathrm{R}$ language, as one of the most fashionable Algorithmic Language now, was applied to analyze the data from GSE25136 and different DEGs compared with previous report was identified. Furthermore, DEGs in PCa recurrence related BP and signaling pathway was screened out, which may help us better understand the potential mechanism of PCa recurrence. Additionally, a PPI network of DEGs was constructed and 5 hub nodes with higher degrees were identified and could be used to predict the prognosis of $\mathrm{PCa}$.

In conclusion, the results of this study provide a comprehensive bioinformatics analysis of DEGs to increase the understading of the mechanism underlying PCa recurrence. The study showed that CD22, IGF-1, INHBA, MAP2K5 and ROR1 may be pivotal for participating in PCa recurrences. However, these functions need to be confirmed by further molecular biological experiments.

\section{References}

1. Hu BR, Fairey AS, Madhav A, Yang D, Li M, Groshen S, Stephens C, Kim PH, Virk N, Wang L, et al: AXIN2 expression predicts prostate cancer recurrence and regulates invasion and tumor growth. Prostate 76: 597-608, 2016.

2. Li HY, Jin N, Han YP and Jin XF: Pathway crosstalk analysis in prostate cancer based on protein-protein network data. Neoplasma 64: 22-31, 2017

3. Stephenson AJ, Scardino PT, Eastham JA, Bianco FJ Jr, Dotan ZA, DiBlasio CJ, Reuther A, Klein EA and Kattan MW: Postoperative nomogram predicting the 10 -year probability of prostate cancer recurrence after radical prostatectomy. J Clin Oncol 23: 7005-7012, 2005 .

4. Hu BR, Fairey AS, Madhav A, Yang D, Li M, Groshen S, Stephens C, Kim PH, Virk N, Wang L, et al: AXIN2 expression predicts prostate cancer recurrence and regulates invasion and tumor growth. Prostate 76: 597-608, 2016.

5. Hao J, Chiang YT, Gout PW and Wang Y: Elevated XPO6 expression as a potential prognostic biomarker for prostate cancer recurrence. Front Biosci (Schol Ed) 8: 44-55, 2016.

6. Choudhury AD, Eeles R, Freedland SJ, Isaacs WB, Pomerantz MM, Schalken JA, Tammela TL and Visakorpi T: The role of genetic markers in the management of prostate cancer. Eur Urol 62: 577-587, 2012.

7. Heidegger I, Höfer J, Luger M, Pichler R, Klocker H, Horninger W, Steiner E, Jochberger S and Culig Z: Is Eotaxin-1 a serum and urinary biomarker for prostate cancer detection and recurrence? Prostate 75: 1904-1909, 2015.
8. Stephenson AJ, Smith A, Kattan MW, Satagopan J, Reuter VE, Scardino PT and Gerald WL: Integration of gene expression profiling and clinical variables to predict prostate carcinoma recurrence after radical prostatectomy. Cancer 104: 290-298, 2005.

9. Sun Y and Goodison S: Optimizing molecular signatures for predicting prostate cancer recurrence. Prostate 69: 1119-1127, 2009.

10. Gene Ontology Consortium: The Gene Ontology (GO) project in 2006. Nucleic Acids Res 34 (Database issue): D322-D326, 2006.

11. Kanehisa M and Goto S. KEGG: Kyoto encyclopedia of genes and genomes. Nucleic Acids Res 28: 27-30, 2000.

12. Thoma C: Prostate cancer: The genomics of localized disease. Nat Rev Urol 14: 65, 2017.

13. Khor LY, Bae K, Al-Saleem T, Hammond EH, Grignon DJ, Sause WT, Pilepich MV, Okunieff PP, Sandler HM and Pollack A: Protein kinase A RI-alpha predicts for prostate cancer outcome: analysis of radiation therapy oncology group trial 86-10. Int J Radiat Oncol Biol Phys 71: 1309-1315, 2008.

14. Govindan R (Ed.): The Washington Manual of Oncology, 2nd edition. Lippincott Williams and Wilkins, Philadelphia, 2008.

15. Merkle D and Hoffmann R: Roles of cAMP and cAMP-dependent protein kinase in the progression of prostate cancer: Cross-talk with the androgen receptor. Cell Signal 23: 507-515, 2011.

16. Rahrmann EP, Collier LS, Knutson TP, Doyal ME, Kuslak SL, Green LE, Malinowski RL, Roethe L, Akagi K, Waknitz M, et al: Identification of PDE4D as a proliferation promoting factor in prostate cancer using a Sleeping Beauty transposon-based somatic mutagenesis screen. Cancer Res 69: 4388-4397, 2009.

17. Henderson DJ, Byrne A, Dulla K, Jenster G, Hoffmann R, Baillie GS and Houslay MD: The cAMP phosphodiesterase-4D7 (PDE4D7) is downregulated in androgen-independent prostate cancer cells and mediates proliferation by compartmentalising cAMP at the plasma membrane of $\mathrm{VCaP}$ prostate cancer cells. $\mathrm{Br}$ J Cancer 110: 1278-1287, 2014.

18. Böttcher R, Henderson DJ, Dulla K, van Strijp D, Waanders LF, Tevz G, Lehman ML, Merkle D, van Leenders GJ, Baillie GS, et al: Human phosphodiesterase 4D7 (PDE4D7) expression is increased in TMPRSS2-ERG-positive primary prostate cancer and independently adds to a reduced risk of post-surgical disease progression. Br J Cancer 113: 1502-1511, 2015.

19. Böttcher R, Dulla K, van Strijp D, Dits N, Verhoef EI, Baillie GS, van Leenders GJ, Houslay MD, Jenster G and Hoffmann R: Human PDE4D isoform composition is deregulated in primary prostate cancer and indicative for disease progression and development of distant metastases. Oncotarget 7: 70669-70684, 2016.

20. Koul HK, Pal M and Koul S: Role of p38 MAP kinase signal transduction in solid tumors. Genes Cancer 4: 342-359, 2013.

21. Guan M, Fousek K and Chow WA: Nelfinavir inhibits regulated intramembrane proteolysis of sterol regulatory element binding protein-1 and activating transcription factor 6 in castration-resistant prostate cancer. FEBS J 279: 2399-2411, 2012.

22. Bach LA and Hale LJ: Insulin-like growth factors and kidney disease. Am J Kidney Dis 65: 327-336, 2015.

23. Shanmugalingam T, Bosco C, Ridley AJ and Van Hemelrijck M: Is there a role for IGF-1 in the development of second primary cancers? Cancer Med 5: 3353-3367, 2016.

24. Cao Y, Nimptsch K, Shui IM, Platz EA, Wu K, Pollak MN, Kenfield SA, Stampfer MJ and Giovannucci EL: Prediagnostic plasma IGFBP-1, IGF-1 and risk of prostate cancer. Int J Cancer 136: 2418-2426, 2015.

25. Dunn SE, Kari FW, French J, Leininger JR, Travlos G, Wilson R and Barrett JC: Dietary restriction reduces insulin-like growth factor I levels, which modulates apoptosis, cell proliferation, and tumor progression in p53-deficient mice. Cancer Res 57: 4667-4672, 1997.

26. McKeown BT and Hurta RA: Magnolol affects expression of IGF-1 and associated binding proteins in human prostate cancer cells in vitro. Anticancer Res 34: 6333-6338, 2014.

27. Shukla S, MacLennan GT, Fu P and Gupta S: Apigenin attenuates insulin-like growth factor-I signaling in an autochthonous mouse prostate cancer model. Pharm Res 29: 1506-2617, 2012.

28. Tsuchiya N, Narita S, Inoue T, Saito M, Numakura K, Huang M, Hatakeyama S, Satoh S, Saito S, Ohyama C, et al: Insulin-like growth factor-1 genotypes and haplotypes influence the survival of prostate cancer patients with bone metastasis at initial diagnosis. BMC Cancer 13: 150, 2013. 
29. Mehta PB, Jenkins BL, McCarthy L, Thilak L, Robson CN Neal DE and Leung HY: MEK5 overexpression is associated with metastatic prostate cancer, and stimulates proliferation, MMP-9 expression and invasion. Oncogene 22: 1381-1389, 2003.

30. MaW,He X,Zhang H,Liu T, Feng X and Zhou Q: Down-regulation of receptor-tyrosine-kinase-like orphan receptor 1 suppresses cell growth and enhances apoptosis in human colorectal carcinoma. Xi Bao Yu Fen Zi Mian Yi Xue Za Zhi 32: 655-665, 2016 (In Chinese)

31. Hojjat-Farsangi M, Moshfegh A, Daneshmanesh AH, Khan AS, Mikaelsson E, Osterborg A and Mellstedt H: The receptor tyrosine kinase ROR1-an oncofetal antigen for targeted cancer therapy. Semin Cancer Biol 29: 21-31, 2014.

32. Oshima T, Yoshihara K, Aoyama T, Hasegawa S, Sato T, Yamamoto N, Akito N, Shiozawa M, Yoshikawa T, Numata K, et al: Relation of INHBA gene expression to outcomes in gastric cancer after curative surgery. Anticancer Res 34: 2303-2309, 2014

33. Lee HY, Li CC, Huang CN, Li WM, Yeh HC, Ke HL, Yang KF, Liang PI, Li CF and Wu WJ: INHBA overexpression indicates poor prognosis in urothelial carcinoma of urinary bladder and upper tract. J Surg Oncol 111: 414-422, 2015
34. Okano M, YamamotoH,Ohkuma H,Kano Y,Kim H,NishikawaS, Konno M, Kawamoto K, Haraguchi N, Takemasa I, et al: Significance of INHBA expression in human colorectal cancer. Oncol Rep 30: 2903-2908, 2013.

35. Tuscano JM, Kato J, Pearson D, Xiong C, Newell L, Ma Y, Gandara DR and O'Donnell RT: CD22 antigen is broadly expressed on lung cancer cells and is a target for antibody-based therapy. Cancer Res 72: 5556-5565, 2012.

36. Pop LM, Barman S, Shao C, Poe JC, Venturi GM, Shelton JM, Pop IV, Gerber DE, Girard L, Liu XY, et al: A reevaluation of CD22 expression in human lung cancer. Cancer Res 74: 263-271, 2014. 